\title{
ON MATHEMATICAL MODELLING OF HEAT AND MOISTURE DISTRIBUTION IN THE DRYING PROCESS FOR POROUS TWO LAYERED GYPSUM BOARD PRODUCTS
}

\author{
Aivars Aboltins ${ }^{1}$, Harijs Kalis ${ }^{2}$, Ilmars Kangro ${ }^{3}$ \\ ${ }^{1}$ Latvia University of Agriculture; ${ }^{2}$ Institute of Mathematics and Computer Science, \\ University of Latvia ${ }^{3}$ Rezekne Academy of Technologies, Latvia \\ aivars.aboltins@1lu.lu,kalis@lanet.lv, ilmars.kangro@ru.lv
}

\begin{abstract}
In this paper we study the problem of the heat and diffusion of one substance through the pores of two layered material of gypsum board products, which may absorb and immobilize some of the diffusing substances with the evolution or absorption of heat. This paper proposes a thermal conductivity model for gypsum plate and gypsum carton at high temperatures, treating gypsum as a porous material consisting of solid and pores. We shall further assume linear dependence on both the temperature $\mathrm{T}$ and the moisture content in every layer $\mathrm{M}=$ const $+\mathrm{aC}-\mathrm{bT}$, where $\mathrm{C}$ is the concentration of water vapour in the air spaces, $\mathrm{M}$ is the amount of moisture absorbed by unit mass of fibre, a and $\mathrm{b}$ are positive constants. For two processes, the transfer of heat and moisture, we derive the system of 4 non-stationary partial differential equations (PDEs), 2 expressing the rate of the change of concentration of water vapour $\mathrm{C}$ in the air spaces and the other 2 the rate of the change of temperature $\mathrm{T}$ in every layer. The approximation of the corresponding initial boundary value problem of the system of PDEs is based on the conservative averaging method (CAM) by using special splines with hyperbolic functions. This procedure allows reducing the 2-D heat and mass transfer initial-boundary problem described by a system of 4 PDEs to initial value problem for a system of 4 ordinary differential equations (ODEs) of the first order. The results of calculations are obtained by MATLAB.
\end{abstract}

Keywords: gypsum board products, heat and diffusion distribution, conservative averaging method, special splines, analytic and numerical solution.

\section{The mathematical model}

The study of heat and mass transfer through a porous media becomes much more interesting due to its vast applications. Many mathematical models are developed for the analysis of such processes, for example, mathematical models of moisture movement in wood, when the wood is considered as porous media.

The study of hydrodynamic flow and heat transfer through a porous media becomes much more interesting due to its vast applications, such as drying in porous solids and soils, drying of wood and paper, soil mechanics, porous heat pipes, paper machines, liquid composite moulding. Many mathematical models are developed for the analysis of such processes, for example mathematical models of moisture movement in wood, when the wood is considered as porous media $[1 ; 2]$.

This paper proposes a thermal conductivity model for gypsum at high temperatures, treating gypsum as a porous material consisting of solid and pores. We study the heat and moisture transfer processes in the two porous layers of gypsum material. We consider the gypsum board material with two layered plates: gypsum plate $(0.0525 \mathrm{~m})$ with density $300 \mathrm{~kg} \cdot \mathrm{m}^{-3}$ and gypsum carton plate $(0.0125 \mathrm{~m})$ with density $1000 \mathrm{~kg} \cdot \mathrm{m}^{-3}$. The gypsum plate on one border is heated with temperature $20+345 \lg (8 t+1)^{\circ} \mathrm{C}$, where $t$ is the time in minutes. In one layer the heat and mass transfer process are analysed and described in $[1 ; 2 ; 5 ; 6]$.

The article [4] has viewed the heating and drying process in wood. In the present article the process of diffusion and heat transfer is consider in 1-D space domain

$$
\Omega=\{(x, y, z): 0 \leq x \leq L,-\infty \leq y \leq \infty,-\infty \leq z \leq \infty\} .
$$

The domain $\Omega$ consists of two layer medium. We will consider the non-stationary problem of the linear diffusion theory for two layered piece-wise homogenous materials in the domain

$$
\Omega_{i}=\left\{(x, y, z): x \in\left(x_{i-1}, x_{i}\right), y \in(-\infty, \infty), z \in(-\infty, \infty)\right\}, i=\overline{1,2},
$$

where $H_{i}=x_{i}-x_{i-1}$ is the height of the layer $\Omega_{i}, x_{0}=0, x_{1}=H_{1}, x_{2}=L=H_{1}+H_{2}$.

We will further assume the linear dependence on both the temperature and moisture content in every layer [1] 


$$
M_{i}=\text { const }+\sigma_{i} C_{i}-\omega_{i} T_{i},
$$

where $C i-$ the concentration of water vapour in the air spaces;

$M_{i}$ - the amount of moisture absorbed by unit mass of fibre;

$\sigma_{i}$ and $\omega_{i}$ are constants.

We will consider the equilibrium uptake of moisture by a fibre to be related to water vapour concentration and temperature $T_{i}$ by the linear relation (1).

Consider an element of a porous material. We can derive two equations, one expressing the rate of the change of concentration and the other - the rate of the change of temperature. The water vapour diffusion PDEs is in the following form

$$
m_{i} g_{i}\left(D_{i} \frac{\partial^{2} C_{i}}{\partial x^{2}}\right)=m_{i} \frac{\partial C_{i}}{\partial t}+\left(1-m_{i}\right) \rho_{s} \frac{\partial M_{i}}{\partial t}: x \in\left[x_{i-1}, x_{i}\right], i=\overline{1,2}, t>0,
$$

where $D_{i}-$ the diffusion coefficient for moisture in air;

$m_{i}-$ is the fraction of the total volume of the material occupied by air;

$\left(1-m_{i}\right)$ - the fraction of the porous material occupied by fibre of density $\rho$;

$t$ - time.

The parameter $g_{i}$ follows from the fact that the diffusion is not along straight air channels. The rate at which the temperature of the element changes is determined by the heat conduction through air and fibres and the heat evolved when moisture is absorbed by fibres. The heat diffusion PDEs can be rewritten in the following form:

$$
c_{i} \rho_{i} \frac{\partial T_{i}}{\partial t}=K_{i} \frac{\partial^{2} T_{i}}{\partial x^{2}}+q_{i} p \frac{\partial M_{i}}{\partial t} ; x \in\left[x_{i-1}, x_{i}\right], i=\overline{1,2}, t>0,
$$

where $c_{i}$ - the specific heat of the fibres;

$K_{i}, \rho_{i}-$ the heat conductivity and the density of the porous material;

$q_{i}-$ the heat evolved when the water vapour is absorbed by the fibres.

We assume that all coefficients in the PDEs are assumed constant and independent of the moisture concentration and temperature.

By eliminating $M_{i}$ from (1), we get the system of four PDEs

$$
\begin{cases}D_{1}^{T} \frac{\partial^{2} T_{1}(x, t)}{\partial x^{2}}=\frac{\partial T_{1}(x, t)}{\partial t}-v_{1} \frac{\partial C_{1}(x, t)}{\partial t}, & D_{2}^{T} \frac{\partial^{2} T_{21}(x, t)}{\partial x^{2}}=\frac{\partial T_{2}(x, t)}{\partial t}-v_{2} \frac{\partial C_{2}(x, t)}{\partial t}, \\ D_{1}^{C} \frac{\partial^{2} C_{1}(x, t)}{\partial x^{2}}=\frac{\partial C_{1}(x, t)}{\partial t}-\lambda_{1} \frac{\partial T_{1}(x, t)}{\partial t}, & D_{2}^{C} \frac{\partial^{2} C_{2}(x, t)}{\partial x^{2}}=\frac{\partial C_{2}(x, t)}{\partial t}-\lambda_{2} \frac{\partial T_{2}(x, t)}{\partial t},\end{cases}
$$

where $\quad D_{i}^{T}=\frac{K_{i}}{\rho_{i}\left(c_{i}+q_{i} \omega_{i}\right)}, D_{i}^{C}=\frac{D_{i} m_{i} g_{i}}{m_{i}+\left(1-m_{i}\right) \rho_{s} \sigma_{i}}, v_{i}=\frac{q_{i} \sigma_{i}}{c_{i}+q_{i} \omega_{i}}$,

$$
\lambda_{i}=\frac{\left(1-m_{i}\right) \omega_{i} \rho_{s}}{m_{i}+\left(1-m_{i}\right) \rho_{s} \sigma_{i}} \lambda_{i} v_{i}<1 \text { are constant coefficients. }
$$

For the initial condition for $t=0$ we give

$$
T_{1}(x, 0)=T_{2}(x, 0)=T_{0}, C_{1}(x, 0)=C_{2}(x, 0)=C_{0},
$$

where $T_{0}, C_{0}-$ known constants.

We use the following boundary and continuous conditions: 


$$
\left\{\begin{array}{l}
D_{1}^{T} \frac{\partial T_{1}(0, t)}{\partial x}-\alpha_{T}\left(T_{1}(0, t)-T_{a}\right)=0, D_{1}^{C} \frac{\partial C_{1}(0, t)}{\partial x}-\alpha_{C}\left(C_{1}(0, t)-C_{a}\right)=0 \\
C_{2}(L, t)=C_{b}, T_{2}(L, t)=T_{b}+T_{l}(t) \\
T_{1}\left(x_{1}, t\right)=T_{2}\left(x_{1}, t\right), D_{1}^{T} \frac{\partial T_{1}\left(x_{1}, t\right)}{\partial x}=D_{2}^{T} \frac{\partial T_{2}\left(x_{1}, t\right)}{\partial x} \\
C_{1}\left(x_{1}, t\right)=C_{2}\left(x_{1}, t\right), D_{1}^{C} \frac{\partial C_{1}\left(x_{1}, t\right)}{\partial x}=D_{2}^{C} \frac{\partial C_{2}\left(x_{1}, t\right)}{\partial x}
\end{array}\right.
$$

where $\alpha_{T}, \alpha_{C}-$ the constant mass transfer coefficients;

$T_{l}(t)=345 \lg (8 t+1)$

$t$ - time in minutes;

$T_{a}, T_{b}, C_{a}, C_{b}$ - the given constants of temperature and concentration on the boundary.

\section{CAM with integral hyperbolic splines in two layers}

The method of conservative averaging by using the special integral splines with hyperbolic trigonometrical functions is openly discussed in papers [8]. We consider the following model problem

$$
\left\{\begin{array}{l}
D_{1}^{T} \frac{\partial T_{1}(0, t)}{\partial x}-\alpha_{T}\left(T_{1}(0, t)-T_{a}\right)=0, D_{1}^{C} \frac{\partial C_{1}(0, t)}{\partial x}-\alpha_{C}\left(C_{1}(0, t)-C_{a}\right)=0, \\
D_{1} \frac{\partial Q_{1}(0, t)}{\partial x}-\alpha_{Q}\left(Q_{1}(0, t)-Q_{a}\right)=0, Q_{2}(L, t)=Q_{C}(t), Q_{1}(x, 0)=Q_{2}(x, 0)=Q_{0}, \\
Q_{1}\left(x_{1}, t\right)=Q_{2}\left(x_{1}, t\right), D_{1} \frac{\partial Q_{1}\left(x_{1}, t\right)}{\partial x}=D_{2} \frac{\partial Q_{2}\left(x_{1}, t\right)}{\partial x},
\end{array}\right.
$$

where $Q=(T, C), T_{c}(t)=T_{b}+T_{l}(t), C_{c}(t)=C_{b}$.

CAM procedure allows reduce the problem to initial problems for the system of ODEs. Using the averaged method with respect to $x$ we have

$$
Q_{i}(x, t)=Q_{i v}(t)+m_{i}(t) \frac{0.5 H_{i} \sinh \left(a_{i}\left(x-\bar{x}_{i}\right)\right)}{\sinh \left(0.5 a_{i} H_{i}\right)}+e_{i}(t)\left(\frac{\cosh \left(a_{i}\left(x-\bar{x}_{i}\right)\right)-A_{i}}{8 \sinh ^{2}\left(0.25 a_{i} H_{i}\right)}\right),
$$

where $Q_{i v}(t)=H_{i}^{-1} \int_{x_{i-1}}^{x_{i}} Q_{i}(x, t) d x, \bar{x}_{i}=\left(x_{i-1}+x_{i}\right) / 2, x \in\left[x_{i-1}, x_{i}\right], A_{i}=\frac{\sinh \left(0.5 a_{i} H_{i}\right)}{0.5 a_{i} H_{i}}, i=1 ; 2$.

We can see, if parameters $a_{1}>0, a_{2}>0$ tend to zero, the limit is the integral parabolic spline (Buikis [7]).

The unknown functions $m_{i}(t), e_{i}(t)$ can be determined from conditions (7):

1) for $x=0 \quad D_{1}\left(m_{1} d_{1}-e_{1} k_{1}\right)-\alpha\left(Q_{1 v}-m_{1} H_{1} / 2+e_{1} b_{1 x}-Q_{a}\right)=0$

2) for $x=L Q_{2 v}+0.5 m_{2} H_{2}+e_{2} b_{2 x}=Q_{c}(t)$

3) for $x=x_{1} Q_{1 v}+0.5 m_{1} H_{1}+e_{1} b_{1 x}=Q_{2 v}-0.5 m_{2} H_{2}+e_{2} b_{2 x}, D_{1}\left(m_{1} d_{1}+e_{1} k_{1}\right)=D_{2}\left(m_{2} d_{2}-e_{2} k_{2}\right)$,

where $d_{i}=0.5 H_{i} a_{i} \operatorname{coth}\left(0.5 a_{i} H_{i}\right)$,

$k_{i}=0.25 a_{i} \operatorname{coth}\left(0.25 a_{i} H_{i}\right)$

$b_{i x}=\frac{\cosh \left(0.5 a_{i} H_{i}\right)-A_{i}}{8 \sinh ^{2}\left(0.25 a_{i} H_{i}\right.}$,

$i=1 ; 2$.

Therefore, we obtain in a similarly way [4] the unknown functions $m_{i}(t), e_{i}(t), i=1 ; 2$.

From (7) follows the system of ODEs: 


$$
\left\{2 D_{1} k_{1} e_{1}(t) / H_{1}=\frac{\partial Q_{1 v}(t)}{\partial t}, \quad 2 D_{2} k_{2} e_{2}(t) / H_{2}=\frac{\partial Q_{2 v}(t)}{\partial t}, Q_{1 v}(0)=Q_{2 v}(0)=Q_{0}\right.
$$

or from (4)

$$
\left\{\begin{array}{l}
\dot{T}_{1 v}=G_{1}\left(b_{11}^{T} T_{1 v}+b_{12}^{T} T_{2 v}+f_{1}^{T}+p_{1}(t)+v_{1}\left(b_{11}^{C} C_{1 v}+b_{12}^{C} C_{2 v}+f_{1}^{C}\right)\right), \\
\dot{T}_{2 v}=G_{2}\left(b_{21}^{T} T_{1 v}+b_{22}^{T} T_{2 v}+f_{2}^{T}+p_{2}(t)+v_{2}\left(b_{21}^{C} C_{1 v}+b_{22}^{C} C_{2 v}+f_{2}^{C}\right)\right), \\
\dot{C}_{1 v}=G_{1}\left(b_{11}^{C} C_{1 v}+b_{12}^{C} C_{2 v}+f_{1}^{C}+\lambda_{1}\left(b_{11}^{T} T_{1 v}+b_{12}^{T} T_{2 v}+f_{1}^{T}+p_{1}(t)\right)\right), \\
\dot{C}_{2 v}=G_{2}\left(b_{21}^{C} C_{1 v}+b_{22}^{C} C_{2 v}+f_{2}^{C}+\lambda_{2}\left(b_{21}^{T} T_{1 v}+b_{22}^{T} T_{2 v}+f_{2}^{T}+p_{2}(t)\right)\right), \\
T_{1 v}(0)=T_{2 v}(0)=T_{0}, C_{1 v}(0)=C_{2 v}(0)=C_{0},
\end{array}\right.
$$

where $b_{i, j}^{T}, b_{i, j}^{C}, f_{i}^{T}, p_{i}(t), f_{i}^{C}$ are known from [4], $G_{i}=\frac{1}{\left(1-\lambda_{i} v_{i}\right)}, i, j=1: 2$.

We rewrite the system of ODEs (11) in the following vector form:

$$
\dot{W}(t)=A W(t)+F+P(t), W(0)=W_{0},
$$

where $W(t), W_{0}, F, P_{0}$ are the 4-order vector-columns with elements

$$
\begin{aligned}
& \left(T_{1 v}(t), T_{2 v}(t), C_{1 v}(t), C_{2 v}(t)\right),\left(T_{0}, T_{0}, C_{0}, C_{0}\right),\left(F_{1}, F_{2}, F_{3}, F_{4}\right),\left(P_{1}, P_{2}, P_{3}, P_{4}\right), \\
& F_{1}=G_{1}\left(f_{1}^{T}+v_{1} f_{1}^{C}\right), F_{2}=G_{2}\left(f_{2}^{T}+v_{2} f_{2}^{C}\right), F_{3}=G_{1}\left(\lambda_{1} f_{1}^{T}+f_{1}^{C}\right), \\
& F_{4}=G_{2}\left(\lambda_{2} f_{2}^{T}+f_{2}^{C}\right), \quad P_{1}(t)=G_{1} p_{1}(t), \quad P_{2}(t)=G_{2} p_{2}(t), \quad P_{3}(t)=G_{1} \lambda_{1} p_{1}(t), \\
& P_{4}(t)=G_{2} \lambda_{2} p_{2}(t),
\end{aligned}
$$

$A$ is the 4-order matrix:

$$
A=\left(\begin{array}{cccc}
G_{1} b_{11}^{T} & G_{1} b_{12}^{T} & G_{1} v_{1} b_{11}^{C} & G_{1} v_{1} b_{12}^{C} \\
G_{2} b_{21}^{T} & G_{2} b_{22}^{T} & G_{2} v_{2} b_{21}^{C} & G_{2} v_{2} b_{22}^{C} \\
G_{1} \lambda_{1} b_{11}^{T} & G_{1} \lambda_{1} b_{12}^{T} & G_{1} b_{11}^{C} & G_{1} b_{12}^{C} \\
G_{2} \lambda_{2} b_{21}^{T} & G_{2} \lambda_{2} b_{22}^{T} & G_{2} b_{21}^{C} & G_{2} b_{22}^{C}
\end{array}\right) .
$$

The averaged solution is

$$
W(t)=W_{1}(t)+W_{2}(t)
$$

where $W_{1}(t)=\exp (A t) W_{0}-(E-\exp (A t)) A^{-1} F \quad$ are the analytical solutions of the ODEs system

$$
\dot{W}_{1}(t)=A W_{1}(t)+F, W_{1}(0)=W_{0} .
$$

Splitting the vector $P(t)$ in the form $P(t)=g(t) P_{0}, P_{0}=\left(G_{1}, G_{2}, G_{1} \lambda_{1}, G_{2} \lambda_{2}\right)$ the solution of the ODEs system $\dot{W}_{2}(t)=A W_{2}(t)+g(t) P_{0}, W_{2}(0)=0, g(t)=T_{l}(t)=345 \lg (8 t+1)$ we can obtain using the spectral decomposition of the matrix A:

$$
\begin{gathered}
A=V D V_{1}, V_{1}=V^{-1}, W_{2}(t)=V R(t), \dot{R}(t)=D R(t)+g(t) V_{1} P_{0}, R(0)=0, \\
R(t)=I(t) V_{1} P_{0}, I(t)=\int_{0}^{t} \exp (D(t-\tau)) g(\tau) d \tau,
\end{gathered}
$$

where $D-$ the diagonal matrix with negative discrete eigenvalues $-k_{i}, i=\overline{1,4}$,

$V$ - the matrix of eigenvectors in the column, $R(t)$ is the column-vector.

If $g(t)=\ln (8 t+1)$ then 


$$
\begin{gathered}
I_{i}(t)=\int_{0}^{t} \exp \left(k_{i}(t-\tau)\right) g(\tau) d \tau= \\
=\frac{1}{\left|k_{i}\right|}\left(\ln (8 t+1)+\exp \left(-(t+1 / 8)\left|k_{i}\right|\right)\left(E_{i}\left(\left|k_{i}\right| / 8\right)-E_{i}\left(\left|k_{i}\right|(1+1 / 8)\right)\right)\right),
\end{gathered}
$$

where $E_{i}(q)-$ the integral exponential ([9], formula 3.352-3 in 325.p).

The solution $W(t)$ can be obtained also with Matlab solver "ode15s".

\section{Some numerical results}

The results of calculations are obtained by MATLAB. We use the discrete values:

$$
\begin{gathered}
x_{j}=j h, j=\overline{0, N}, N H=L=0.065 m, H_{1}=0.0525 m, H_{2}=0.0125 m, t_{n}=n \tau, \\
n=\overline{0, N_{t}}, N_{t} \tau=t_{f}=1000 \mathrm{~s}, N_{t}=50, T_{0}=20^{\circ} \mathrm{C}, C_{0}=2{ }^{\circ} \mathrm{C}, T_{a}=20^{\circ} \mathrm{C}, \\
T_{b}=20+345 \lg (8 t+1)^{\circ} \mathrm{C}, C_{a}=2{ }^{\circ} \mathrm{C}, C_{b}=0{ }^{\circ} \mathrm{C} .
\end{gathered}
$$

We use the following parameters in each layer (such as the value of the parameter $\rho$ in the first layer is 300 , and the second layer is 1000 ):

$$
\begin{gathered}
\rho=[300 ; 1000] \frac{\mathrm{kg}^{3}}{\mathrm{~m}^{3}}, K=[0.4 ; 0.8] \frac{\mathrm{W}}{\mathrm{m} \cdot{ }^{0} \mathrm{C}}, D=\left[0.1 \cdot 10^{-6} ; 0.80 \cdot 10^{-6}\right] \frac{\mathrm{m}}{\mathrm{s}^{2}}, \sigma=[0.8 ; 0.4], \\
\omega=[0.8 ; 1.0], g=[1 ; 1], q=[0.8 ; 0.9], m=[0.7 ; 0.9], c_{p}=[800 ; 700] \frac{\mathrm{J}}{\mathrm{m}}, \alpha=[0.01 ; 0.1], \rho_{s}=1 .
\end{gathered}
$$

The results of calculation are represented in Table 1 and Figs. (1-8), where $T_{1 v}, T_{2 v}$ are the averaging values for temperature in layers, $t_{f}$ the final time in sec. The parameters $a_{1}=20, a_{2}=10$ are obtained for minimal value of maximal error for averaging values.

Table 1

The values of $t_{f}, T\left(0, t_{f}\right) T\left(H_{1}, t_{f}\right), T\left(H_{1}, t_{f}\right), T_{1 v}\left(t_{f}\right), T_{2 v}\left(t_{f}\right), \max C\left(x, t_{f}\right)$

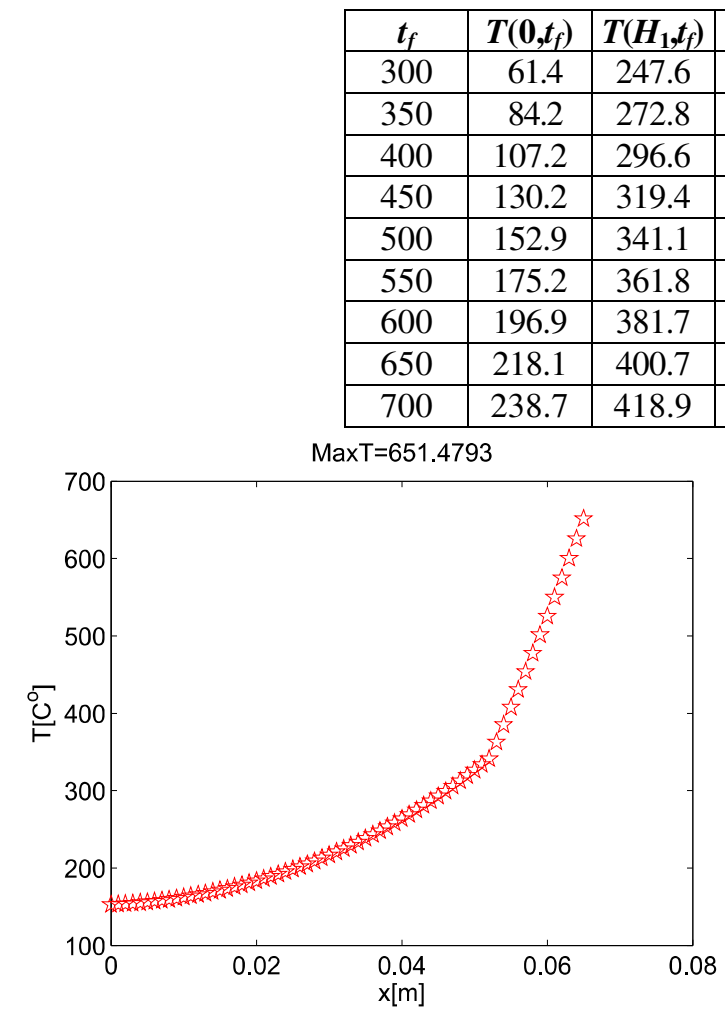

Fig. 1. Temperature depending on $x$ for $t_{f}=500 \mathrm{~s}$

\begin{tabular}{|c|c|c|c|}
\hline$\left(\boldsymbol{L}, \boldsymbol{t}_{\boldsymbol{f}}\right)$ & $\boldsymbol{T}_{\mathbf{1 v}}\left(\boldsymbol{t}_{\boldsymbol{f}}\right)$ & $\boldsymbol{T}_{\mathbf{2 v}}\left(\boldsymbol{t}_{f}\right)$ & $\max \boldsymbol{C}\left(\boldsymbol{x}, \boldsymbol{t}_{f}\right)$ \\
\hline 576.4 & 122.4 & 405.6 & 0.55 \\
\hline 598.9 & 146.5 & 430.1 & 0.41 \\
618.6 & 170.4 & 452.4 & 0.31 \\
635.9 & 193.9 & 472.8 & 0.24 \\
651.5 & 216.8 & 491.8 & 0.22 \\
665.6 & 239.2 & 509.5 & 0.19 \\
\hline 678.4 & 260.9 & 526.1 & 0.16 \\
\hline 690.3 & 281.9 & 541.7 & 0.13 \\
701.3 & 302.3 & 556.5 & 0.10 \\
\hline
\end{tabular}

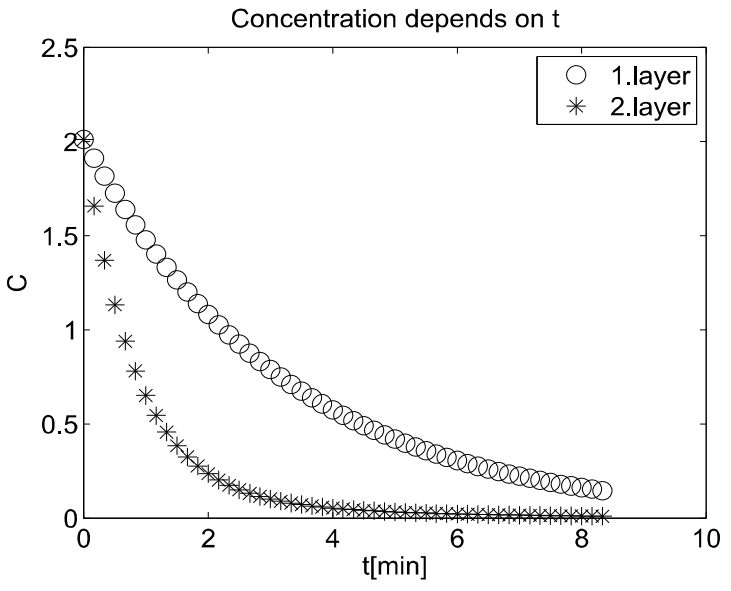

Fig. 2. Averaging concentration depending on $t$ for $t_{f}=500 \mathrm{~s}$ 


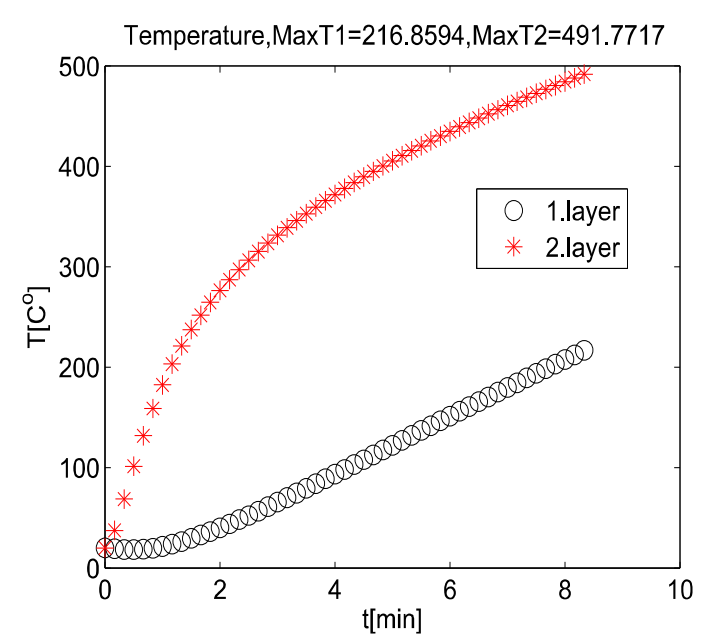

Fig. 3. Averaging temperature depending on $t$ for $t_{f}=500 \mathrm{~s}$

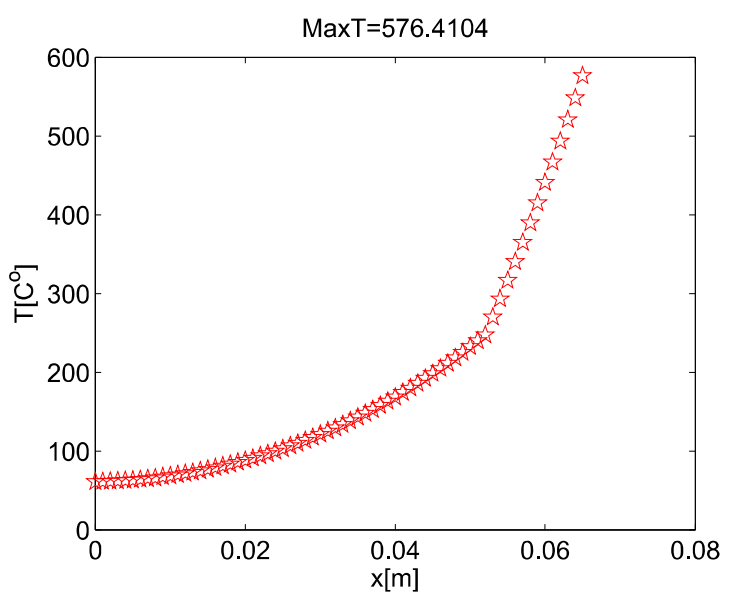

Fig. 5. Temperature depending on $x$ for $t_{f}=300 \mathrm{~s}$

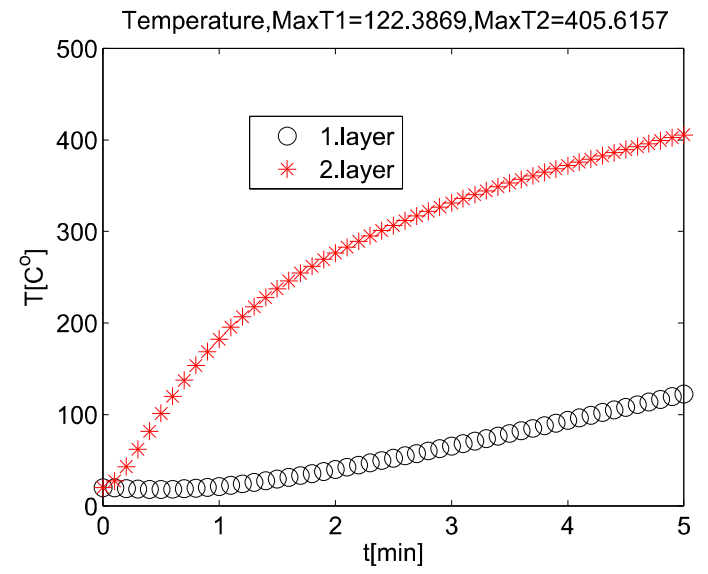

Fig.7. Averaging temperature depending on $\boldsymbol{t}$ for $t_{f}=300 \mathrm{~s}$

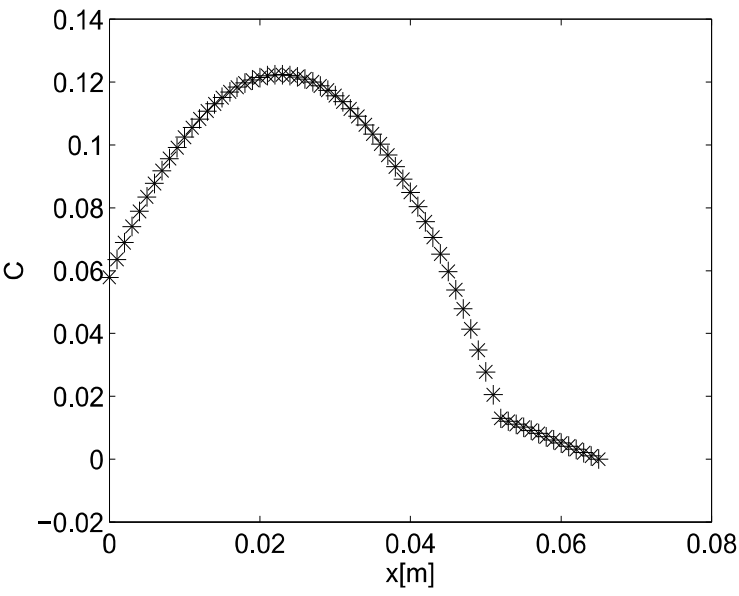

Fig. 4. Concentration depending on $x$ for $t_{f}=500 \mathrm{~s}$

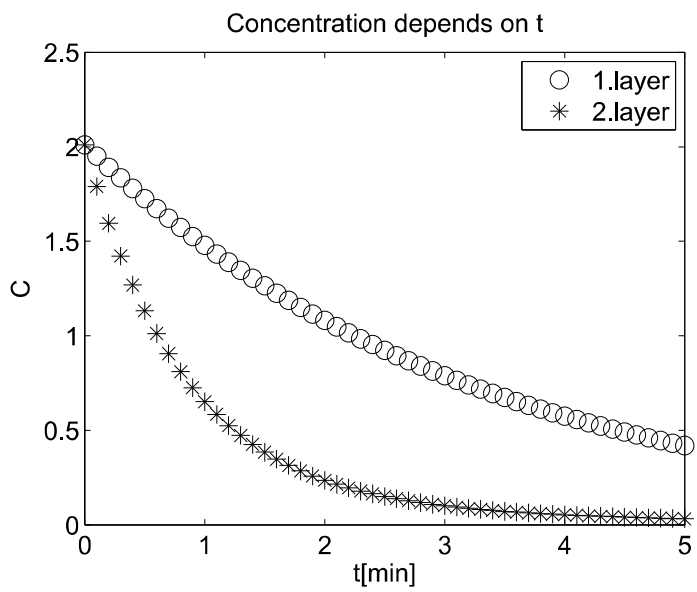

Fig. 6. Averaging concentration depending on $t$ for $t_{f}=300 \mathrm{~s}$

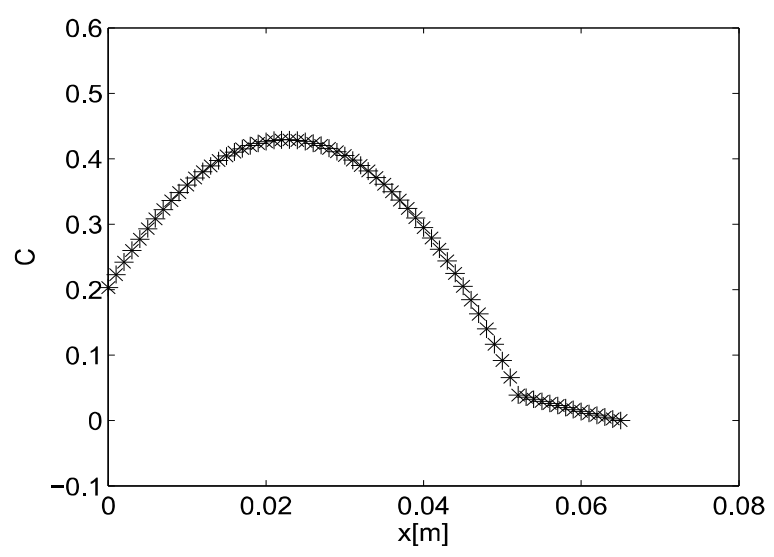

Fig. 8. Concentration depending on $x$ for $t_{f}=300 \mathrm{~s}$

For the numerical experiment we use also backward orientation: for gypsum plate $H_{2}=0.0525 \mathrm{~m}$ with density $\rho_{2}=300 \mathrm{~kg} \cdot \mathrm{m}^{-3}$ gypsum carton plate $H_{2}=0.0125 \mathrm{~m}$ with density $\rho_{1}=1000 \mathrm{~kg} \cdot \mathrm{m}^{-3}$, and

$$
K=[0.8 ; 0.4] \frac{W}{m \cdot{ }^{\circ} C}, D=\left[0.8 \cdot 10^{-6} ; 0.1 \cdot 10^{-6}\right] \frac{m}{s^{2}}, \sigma=[0.4 ; 0.8],
$$




$$
\omega=[1.0 ; 0.8], g=[1 ; 1], q=[0.9 ; 0.8], m=[0.9 ; 0.7], c_{p}=[700 ; 800] \frac{J}{m}, \alpha=[0.1 ; 0.01], \rho_{s}=1 \text {. }
$$

The results of calculation are represented in Table 2 and Fig. (9). The distribution of temperature is nearly linear, the temperature increase in $x=0$ and the averaging temperature decrease in second layer.

The values of $t_{f}, T\left(0, t_{f}\right) T\left(H_{1}, t_{f}\right), T\left(H_{1}, t_{f}\right), T_{1 v}\left(t_{f}\right), T_{2 v}\left(t_{f}\right)$

Table 2

\begin{tabular}{|c|c|c|c|c|c|}
\hline $\boldsymbol{t}_{f}$ & $\boldsymbol{T}\left(\mathbf{0}, \boldsymbol{t}_{f}\right)$ & $\boldsymbol{T}\left(\boldsymbol{H}_{\mathbf{1}}, \boldsymbol{t}_{f}\right)$ & $\boldsymbol{T}\left(\boldsymbol{L}, \boldsymbol{t}_{f}\right)$ & $\boldsymbol{T}_{\mathbf{1 v}}\left(\boldsymbol{t}_{f}\right)$ & $\boldsymbol{T}_{\mathbf{2 v}}\left(\boldsymbol{t}_{f}\right)$ \\
\hline 300 & 76.9 & 141.6 & 576.4 & 99.50 & 309.1 \\
\hline 350 & 114.2 & 182.7 & 598.9 & 138.8 & 345.0 \\
\hline 400 & 151.2 & 221.9 & 618.6 & 177.2 & 378.0 \\
\hline 450 & 186.9 & 259.1 & 635.9 & 234.1 & 408.5 \\
\hline 500 & 220.9 & 293.9 & 651.5 & 249.0 & 436.7 \\
\hline 550 & 252.9 & 326.5 & 665.6 & 281.8 & 462.7 \\
\hline 600 & 282.9 & 356.8 & 678.4 & 312.6 & 486.8 \\
\hline 650 & 311.0 & 385.1 & 690.3 & 341.2 & 509.1 \\
\hline 700 & 337.2 & 411.3 & 701.3 & 367.9 & 529.8 \\
\hline \multicolumn{5}{|c|}{ MaxT=651.4793 } \\
\hline
\end{tabular}

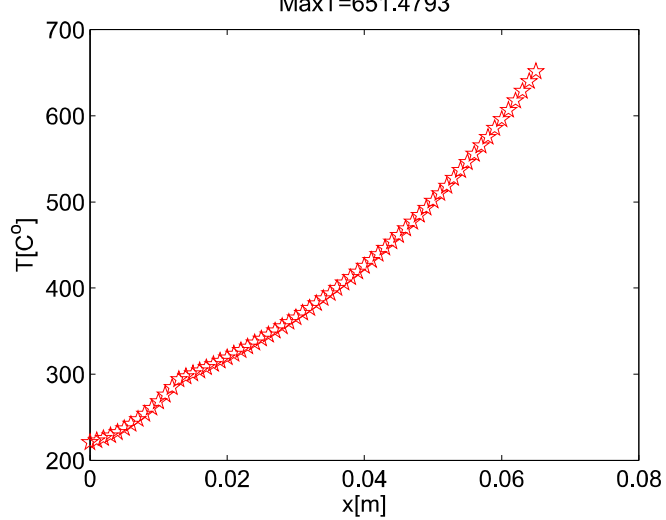

Fig. 9. Backward temperature depending on $x$ for $t_{f}=300 \mathrm{~s}$

\section{Conclusions}

1. For the transfer of heat in porous layer is considered the system of 4 PDEs for determination the concentration $C$ of water vapour in the air spaces and the temperature $T$.

2. The approximation of the corresponding initial boundary value problem of the system of PDEs is based on the conservative averaging method (CAM).

3. CAM is used with new hyperbolic type splines. For these splines the best parameter for minimal error is calculated. This method can be used for solution of multi dimension 3D problem of PDEs.

4. The problem of the system of PDEs with constant coefficients is approximated on the initial value problem of the system of ODEs of the first order, where it is solved analytically and with Matlab solvers.

5. Such a procedure allows us to obtain a simple engineering algorithm for solving mass transfer equations for different substances in layered domain.

6. The results of the numerical experiments can give some new physical conclusions about the drying process in porous material.

\section{Acknowledgments}

This work was partially supported by the grant 623/2014 of the Latvian Council of Science.

\section{References}

1. Crank J. The mathematics of diffusion. Oxford, at the Clarendon Press, 1956. 
2. Kalis H., Kangro I. Calculation of heat and moisture sistribution in the porous media layer. Mathematical Modelling and Analysis, vol. 12, No. 1, 2007, pp. 91-100.

3. Kalis H., Kangro I. Simple methods of engineering calculation for solving multi-substances transfer problem in multi-layer media. Mathematical Modelling and Analysis, vol. 8, No. 1,2006, pp. 33-42.

4. Aboltins A., Kalis H., Kangro I. On mathematical modelling of heat and moisture distribution in porous multilayer media. Proc. of 15-th int. conf. for Rural Development, Jelgava, 25-27.05.2016, pp. 37-44.

5. Rahmanian I., Wang Y.C. A combined experimental and numerical method for extracting temperature-dependent thermal conductivity of gypsum boards. Construction and Building Materials 26, 2012, pp. 707-722.

6. Weber B. Heat transfer mechanisms and models for a gypsum board exposed to fire. International Journal of Heat and Mass Transfer 55, 2012, pp. 1661-1678.

7. Buike M., Buikis A. Modelling 3-D transport processes in anisotropic layered stratum by conservative averaging method. WSEAS Transactions on Heat and Mass Transfer, 2006, vol.1, Nr. 4, pp. 430-437.

8. Buikis A., Kalis H., Kangro I. Special Hyperbolic type spline for mass transfer problems in multilayer 3-D domains. Mathematical and Computational Methods in Applied Sciences. Proc. of the 3-rd int. conf. on Applied, Numerical and Cmputational Mathematics (ICANCM'15), Sliema, Malta,aug.17-19,2015, pp. 25-34.

9. Gradshtein I.S., Ryhzik I.M. Table of integrals, summ, series and multiplicatives. Nauka Press, Moscow, 1962., in Russian. 